\title{
Teores foliares de fósforo e zinco, produtividade e crescimento de café irrigado
}

\author{
Myriane Stella Scalco(1), Livia Alves Alvarenga(1), Rubens José Guimarães(2), Anderson Willian Dominghetti(2), \\ Alberto Colombo(1), Gleice Aparecida Assis( ${ }^{(3)}$ e Giselle Figueiredo Abreu ${ }^{(1)}$
}

\begin{abstract}
(1)Universidade Federal de Lavras (Ufla), Departamento de Engenharia, Caixa Postal 3.037, CEP 37200-000 Lavras, MG, Brasil. E-mail: msscalco@dag.ufla.br, liviaalvesalvarenga@yahoo.com.br, acolombo@deg.ufla.br, gfigueiredoabreu@agronomia.ufla.br (2)Ufla, Departamento de Agricultura, Caixa Postal 3.037, CEP 37200-000 Lavras, MG, Brasil. E-mail: rubensjg@dag.ufla.br, andersonwd10@yahoo.com.br (3)Universidade Federal de Uberlândia, Instituto de Ciências Agrárias, Campus Monte Carmelo, Caixa Postal 5, CEP 38500-000 Monte Carmelo, MG, Brasil. E-mail: gleice_ufla@yahoo.com.br
\end{abstract}

Resumo - O objetivo deste trabalho foi avaliar os efeitos da irrigação sobre as concentrações foliares de P e Zn e sobre a produtividade e o crescimento do cafeeiro (Coffea arabica), cultivado em plantio tradicional e adensado. Utilizou-se o delineamento experimental de blocos ao acaso, em arranjo de parcelas subdivididas, com quatro repetições. As parcelas corresponderam às densidades de plantio (3.333 e 10.000 plantas ha $\left.\mathrm{a}^{-1}\right)$, e as subparcelas corresponderam às lâminas d'água, aplicadas por quatro manejos de irrigação: início da irrigação, quando a tensão d'água no solo à profundidade de $0,25 \mathrm{~m}$ atingia valores próximos a 20 ou $60 \mathrm{kPa}$; turno fixo de irrigação (três vezes por semana), de acordo com o balanço hídrico climatológico; e testemunha sem irrigação. Em 2009, 2010 e 2011, foram feitas as seguintes avaliações: teores foliares de P e Zn; produtividade de café beneficiado (sacas ha-1); e área lateral do dossel. Lâminas de irrigação maiores favorecem a absorção radicular de $\mathrm{P}$ e o crescimento vegetativo das plantas no sistema tradicional e no adensado, bem como o aumento da produtividade no sistema tradicional. $\mathrm{O}$ aumento da disponibilidade de $\mathrm{P}$ para as plantas, pelo aumento da irrigação, diminui a concentração de Zn nas folhas.

Termos para indexação: Coffea arabica, densidade de plantio, estado nutricional, fertirrigação, interação entre nutrientes.

\section{Leaf contents of phosphorus and zinc, productivity, and growth of irrigated coffee}

\begin{abstract}
The objective of this work was to evaluate the effects of irrigation on leaf contents of $\mathrm{P}$ and $\mathrm{Zn}$, on the yield, and on the growth of coffee plants (Coffea arabica) under traditional and high density planting. A randomized complete block design was used in split-plot arrangement, with four replicates. Plots consisted of planting density $\left(3,333\right.$ and 10,000 plants ha $\left.{ }^{-1}\right)$, and subplots consisted of water depths applied according to four irrigation managements: beginning of irrigation whenever water tension at $0.25 \mathrm{~m}$ soil depth reached values near 20 or $60 \mathrm{kPa}$; fixed irrigation turn (three times a week), according to climatic water balance; and a control without irrigation. In 2009, 2010, and 2011, the following evaluations were done for: leaf concentrations of $\mathrm{P}$ and $\mathrm{Zn}$; yield of processed coffee (bags ha ${ }^{-1}$ ); and lateral canopy area. Larger water irrigation depths favor root absorption of $\mathrm{P}$ and the vegetative growth of plants, on both traditional and high density planting systems, as well as productivity increase in the traditional system. Phosphorus increased availability for plants by greater irrigation depths decreases $\mathrm{Zn}$ concentration in the leaves.
\end{abstract}

Index terms: Coffea arabica, planting density, nutritional status, fertigation, nutrient interaction.

\section{Introdução}

A irrigação favorece significativamente 0 desenvolvimento vegetativo (Miranda et al., 2011) e a produtividade do cafeeiro (Coffea arabica L.) (Silva et al., 2008; Scalco et al., 2011), e o aumento de eficiência dos fertilizantes é uma das principais vantagens da fertirrigação (Guimarães et al., 2010;
Rezende et al., 2010; Sobreira et al., 2011). No entanto, ainda há dificuldades no manejo da irrigação e dos fertilizantes em lavouras cafeeiras, além de carência de informações sobre a nutrição de plantas irrigadas de café (Fernandez et al., 2007).

$\mathrm{O}$ aumento da eficiência nutricional das plantas pode ter grande impacto sobre a produtividade do cafeeiro e seus custos de produção (Amaral et al., 
2011). Especificamente quanto ao fósforo, o consumo médio em cafezais brasileiros pode chegar a 164.611 toneladas de $\mathrm{P}_{2} \mathrm{O}_{5}$ por ano (Guimarães et al., 2010), e a irrigação pode otimizar o uso deste fertilizante e proporcionar grande economia para os cafeicultores. A disponibilidade de água tem forte influência sobre a absorção de $\mathrm{P}$ pelas plantas, pois seu conteúdo no solo age sobre a difusão do nutriente (Aquino et al., 2012).

No entanto, o fósforo tem relação antagônica com Zn e, portanto, cuidados com o equilíbrio entre nutrientes devem ser tomados (Politi et al., 2013). Trabalhos têm sido realizados para um maior entendimento da interação entre $\mathrm{P}$ e $\mathrm{Zn}$, com uso de altas doses de $\mathrm{P}$ (Reis Junior \& Martinez, 2002); porém, ainda faltam informações sobre cultivos irrigados de café.

Além disso, como o arranjo das plantas a diferentes densidades de plantio pode alterar a produtividade do cafeeiro (Pereira et al., 2011), é importante que se busquem respostas quanto à absorção de nutrientes em sistemas irrigados de cultivo adensado e tradicional, pois estas certamente serão diferentes das obtidas em cultivos de sequeiro.

O objetivo deste trabalho foi avaliar os efeitos da irrigação sobre as concentrações foliares de $\mathrm{P}$ e $\mathrm{Zn}$ e sobre a produtividade e o crescimento do cafeeiro (Coffea arabica), cultivado em plantio tradicional e adensado.

\section{Material e Métodos}

O experimento foi realizado no Município de Lavras, MG $\left(21^{\circ} 14^{\prime} 06^{\prime \prime} \mathrm{S}, 45^{\circ} 00^{\prime} 00^{\prime \prime} \mathrm{W}\right.$, à altitude de $\left.910 \mathrm{~m}\right)$, no setor de cafeicultura do Departamento de Agricultura, Universidade Federal de Lavras, de 2009 a 2011. O clima, conforme a classificação de Köppen, é do tipo Cwa, mesotérmico com verões brandos, e estiagem de inverno. A precipitação e a temperatura média anual são de $1.460 \mathrm{~mm}$ e $20,4^{\circ} \mathrm{C}$, respectivamente (Dantas et al., 2007).

O solo da área experimental é um Latossolo Vermelho distroférrico (Santos et al., 2006) de textura argilosa, e suas características químicas, na camada de 0 a $20 \mathrm{~cm}$ de profundidade, estão apresentadas na Tabela 1 .

A lavoura utilizada era da cultivar Rubi MG 1192, com seis anos de idade. Ela foi podada (esqueletamento a $0,40 \mathrm{~m}$ ) e decotada (a 1,40 m) em 2007. As adubações foram realizadas conforme Guimarães et al. (1999), de acordo com a análise de solo, enquanto a correção foi feita com o acréscimo de $30 \%$ da dose de calcário, tendo-se seguido a recomendação para cafeeiros irrigados (Santinato \& Fernandes, 2002). Nos anos de avaliação, as adubações foram parceladas em oito vezes, de outubro a janeiro, e realizadas via fertirrigação nas parcelas irrigadas, e a lanço (adubação manual) nas parcelas não irrigadas. Como fontes de nitrogênio, foram utilizadas a ureia pecuária $(45 \%$ de $\mathrm{N})$ e o nitrato de potássio $(13 \%$ de $\mathrm{N})$, que também forneceu potássio $\left(47 \%\right.$ de $\left.\mathrm{K}_{2} \mathrm{O}\right)$. Em 2010, aplicaram-se $20 \mathrm{~g}$ de $\mathrm{P}_{2} \mathrm{O}_{5}$ por cova, tendo-se como fonte o MAP (54\% de $\mathrm{P}_{2} \mathrm{O}_{5}$ e $9 \%$ de $\mathrm{N}$ ), que também foi contabilizado na reposição de N. Os micronutrientes foram fornecidos via foliar, à concentração de $0,3 \%$ de ácido bórico, $0,3 \%$ de sulfato de zinco e $0,3 \%$ de cloreto de potássio. Em 2009, aplicaram-se $2 \mathrm{Mg} \mathrm{ha}^{-1}$ de calcário, conforme Guimarães et al. (1999).

Utilizou-se o delineamento experimental de blocos ao acaso, em arranjo de parcelas subdivididas, com quatro repetições, em que as parcelas consistiram da densidade de plantio, e as subparcelas das lâminas de irrigação em cada manejo. Cada subparcela foi composta por dez plantas, tendo-se considerado as oito centrais como úteis.

Avaliaram-se as densidades de plantio de 3.333 plantas ha-1 $(3 \times 1 \mathrm{~m})$ e de 10.000 plantas $^{-1}$

Tabela 1. Análise química do solo da área experimental $(0-20 \mathrm{~cm})$, nos três anos de avaliação ${ }^{(1)}$.

\begin{tabular}{|c|c|c|c|}
\hline \multirow[t]{2}{*}{ Característica } & \multicolumn{3}{|c|}{ Ano } \\
\hline & 2008 & 2009 & 2010 \\
\hline pH em água & 5,1 & 5,1 & 5,1 \\
\hline P-Mehlich $1\left(\mathrm{mg} \mathrm{dm}^{-3}\right)$ & 48,9 & 40,0 & 45,3 \\
\hline P-remanescente $\left(\mathrm{mg} \mathrm{L}^{-1}\right)$ & 19,8 & 14,3 & 15,1 \\
\hline $\mathrm{K}\left(\mathrm{mg} \mathrm{dm}{ }^{-3}\right)$ & 113,3 & 154,7 & 169,0 \\
\hline $\mathrm{Ca}\left(\mathrm{cmol}_{\mathrm{c}} \mathrm{dm}^{3}\right)$ & 1,8 & 1,8 & 2,5 \\
\hline $\mathrm{Mg}\left(\mathrm{cmol}_{\mathrm{c}} \mathrm{dm}^{-3}\right)$ & 0,7 & 0,6 & 1,0 \\
\hline $\mathrm{Al}\left(\mathrm{cmol}_{\mathrm{c}} \mathrm{dm}^{-3}\right)$ & 0,5 & 0,5 & 0,3 \\
\hline $\mathrm{H}+\mathrm{Al}\left(\mathrm{cmol}_{\mathrm{c}} \mathrm{dm}^{-3}\right)$ & 7,3 & 6,9 & 5,8 \\
\hline Boro $\left(\mathrm{mg} \mathrm{dm}^{-3}\right)$ & 0,4 & 0,4 & 0,3 \\
\hline Zinco $\left(\mathrm{mg} \mathrm{dm}^{-3}\right)$ & 11,5 & 9,4 & 10,1 \\
\hline Cobre $\left(\mathrm{mg} \mathrm{dm}^{-3}\right)$ & 2,5 & 2,7 & 2,9 \\
\hline $\operatorname{Mn}\left(\mathrm{mg} \mathrm{dm}^{-3}\right)$ & 7,1 & 8,4 & 12,2 \\
\hline Ferro $\left(\mathrm{mg} \mathrm{dm}^{-3}\right)$ & 42,7 & 42,9 & 48,2 \\
\hline $\mathrm{S} \mathrm{B}\left(\mathrm{cmol}_{\mathrm{c}} \mathrm{dm}^{-3}\right)$ & 2,8 & 2,9 & 4,0 \\
\hline $\mathrm{t}\left(\mathrm{cmol}_{\mathrm{c}} \mathrm{dm}^{-3}\right)$ & 3,3 & 3,4 & 4,1 \\
\hline $\mathrm{T}\left(\mathrm{cmol}_{\mathrm{c}} \mathrm{dm}^{-3}\right)$ & 10,2 & 9,8 & 9,8 \\
\hline $\mathrm{m}(\%)$ & 16,0 & 12,8 & 5,4 \\
\hline $\mathrm{V}(\%)$ & 29,2 & 29,5 & 40,1 \\
\hline $\operatorname{MOS}($ dag kg-1) & 2,8 & 3,7 & 3,2 \\
\hline
\end{tabular}

$\mathrm{SB}$, soma de bases; t, CTC efetiva; T, CTC a pH 7,0; m, saturação por alumínio; V, saturação por bases; MOS, matéria orgânica do solo. 
(2,0x0,5 m) e os seguintes manejos de irrigação: irrigações sempre que a tensão da água no solo, à profundidade de $0,25 \mathrm{~m}$, atingia valores próximos de $60 \mathrm{kPa}$ (turno de irrigação variável); irrigações sempre que a tensão da água no solo, à profundidade de $0,25 \mathrm{~m}$, atingia valores próximos de $20 \mathrm{kPa}$ (turno de irrigação variável); irrigações em turnos fixos, três vezes por semana (segunda, quarta e sexta-feira), de acordo com o balanço hídrico climatológico (BHC); e um tratamento testemunha não irrigado.

Nas parcelas irrigadas com base na tensão de água no solo, o manejo da irrigação foi feito por meio de tensiômetros, cujas leituras foram feitas em um tensímetro de punção digital. Os tensiômetros foram instalados às profundidades de 10,25, 40 e $60 \mathrm{~cm}$, e o momento de irrigar foi definido pelo tensiômetro à profundidade de $25 \mathrm{~cm}$. Para cálculo das lâminas aplicadas, foram consideradas as tensões registradas nas quatro profundidades de instalação dos tensiômetros. Esses valores de tensão foram convertidos em umidade volumétrica, por meio de curvas de retenção de água do solo, ajustadas pelo modelo de Van Genutchen (1980), para as camadas de 0-20, 20-40 e 30-60 cm de profundidade (Serra et al., 2013).

O BHC foi utilizado para definir o volume de aplicação para o manejo com calendário fixo de irrigação. As lâminas aplicadas neste tratamento foram calculadas de acordo com os valores de precipitação pluvial efetiva, de evapotranspiração da cultura (ETc) e de percolação para além da profundidade efetiva do sistema radicular $(60 \mathrm{~cm})$. O BHC foi efetuado com o programa Irriplus (Centev/UFV,Viçosa, MG), com dados diários de: temperaturas máxima, média e mínima; velocidade do vento; umidade relativa do ar; radiação solar; e precipitação. Esses dados foram obtidos de uma estação meteorológica automática, umetos (Pessl Instruments, Ltd., Weiz, Áustria), instalada na área experimental. A evapotranspiração da cultura (Etc) foi calculada a partir da evapotranspiração de referência (ETo) pelo método de Penman-Monteith (Allen et al., 1998), corrigida pelos coeficientes de deficit diário de umidade do solo (Ks), pelo coeficiente de cultura $(\mathrm{Kc}=0,75)$ e pelo coeficiente de localização (K1), conforme descrito por Mantovani et al. (2007). O sistema de irrigação por gotejamento foi composto por linhas laterais com gotejadores autocompensantes, de vazão nominal de $3,75 \mathrm{~L}$ por hora, espaçados de $40 \mathrm{~cm}$, que formaram uma faixa molhada ao longo da linha de plantas.
Nos anos de 2009, 2010 e 2011, foram avaliados: em janeiro, os teores de $\mathrm{P}$ e $\mathrm{Zn}$ em folhas amostradas do terço médio de oito plantas úteis, de cada subparcela experimental; em julho, as produtividades de café beneficiado (sacas $\mathrm{ha}^{-1}$ ); e, ao final de dezembro - última avaliação de crescimento - a área foliar lateral do dossel, estimada conforme Favarin et al. (2002) As lâminas aplicadas em cada manejo de irrigação e as precipitações consideradas na avaliação corresponderam aos períodos de janeiro a dezembro de 2008, 2009 e 2010 (Tabela 2).

Utilizou-se a correlação linear de Pearson para avaliar o grau de relacionamento entre as seguintes variáveis: teores de $\mathrm{P}$ e $\mathrm{Zn}$ nas folhas; área lateral do dossel $\left(\mathrm{m}^{2}\right)$; produtividade de café beneficiado (sacas ha-1); e lâminas de irrigação aplicadas em cada ano de avaliação. O modelo de regressão foi utilizado para descrever o comportamento do teor foliar de $\mathrm{P}$ e Zn, em razão das lâminas aplicadas a cada ano, nas situações em que os desdobramentos das interações entre esses fatores foram pertinentes.

\section{Resultados e Discussão}

Observou-se correlação positiva e significativa entre a lâmina de irrigação aplicada e os teores foliares de $\mathrm{P}$, nas duas densidades avaliadas, durante os três anos de estudo (Tabela 3). Este resultado evidencia o potencial da fertirrigação para a melhoria do aproveitamento do fertilizante fosfatado (Rezende et al., 2010) e indica que a reavaliação das doses de aplicação de $\mathrm{P}$ pode ser pertinente em lavouras irrigadas, uma vez que a quantidade de água aplicada interferiu positivamente sobre a quantidade de $\mathrm{P}$ absorvida pelas plantas. A movimentação de $\mathrm{P}$ no solo se dá pelo processo de difusão, que tem a água como principal veículo. Portanto, o aumento na disponibilidade hídrica do solo favorece a absorção deste nutriente.

Tabela 2. Lâminas d'água (mm) aplicadas de acordo com os diferentes manejos de irrigação nas densidades de 3.333 e 10.000 plantas por hectare.

\begin{tabular}{lcccccccc}
\hline Ano & \multicolumn{3}{c}{3.333 plantas ha-1 } & & \multicolumn{3}{c}{10.000 plantas ha $^{-1}$} & Precipitação \\
\cline { 2 - 3 } & $60 \mathrm{kPa}$ & $20 \mathrm{kPa}$ & BHC & & $60 \mathrm{kPa}$ & $20 \mathrm{kPa}$ & $\mathrm{BHC}$ & $(\mathrm{mm})$ \\
\hline 2008 & 195,3 & 353,0 & 254,9 & & 254,7 & 561,1 & 405,5 & $1.586,4$ \\
2009 & 195,2 & 292,2 & 523,8 & & 264,6 & 596,2 & 639,6 & $1.812,0$ \\
2010 & 284,0 & 344,2 & 412,5 & & 460,3 & 687,2 & 514,6 & $1.248,0$ \\
\hline Média & 224,8 & 329,8 & 397,1 & & 326,5 & 614,8 & 519,9 & $1.548,8$ \\
\hline
\end{tabular}


As lâminas de irrigação também se correlacionaram significativamente com a área lateral do dossel, nas duas densidades e nos três anos. No entanto, entre os teores foliares de $\mathrm{P}$ e a área lateral, somente não se observou correlação significativa em 2011, à densidade de 10.000 plantas ha ${ }^{-1}$. Portanto, o aumento da disponibilidade de água favoreceu a absorção de $\mathrm{P}$ que, por sua vez, favoreceu o crescimento da parte aérea, o que está de acordo com Aquino et al. (2012).

Guerra et al. (2007) observaram respostas lineares da produtividade do cafeeiro à aplicação de fósforo até $400 \mathrm{~kg} \mathrm{ha}^{-1}$ de $\mathrm{P}_{2} \mathrm{O}_{5}$, em lavouras irrigadas, o que indica que esta prática pode aumentar a resposta das plantas a doses maiores de adubação fosfatada. De maneira semelhante, Mera et al. (2011) relataram interação entre regime hídrico e doses de $\mathrm{P}_{2} \mathrm{O}_{5}$ em algumas variáveis de crescimento; porém, os autores apontaram a necessidade de novos estudos, com aplicação de maiores doses de fósforo, para que se encontre um ponto de inflexão na curva de produtividade do cafeeiro em resposta à adubação com $\mathrm{P}$

No presente trabalho, a frequência com que a produtividade correlacionou-se significativamente com os teores de $\mathrm{P}$ ou com as lâminas de irrigação não foi alta. Nos anos de 2009 e 2011, de alta produtividade, essas correlações foram positivas e significativas, à densidade de plantas de 3.333 ha $^{-1}$ (Tabela 3), possivelmente em razão da maior necessidade de $\mathrm{P}$ nos anos de maior produtividade $\left(67,85 \mathrm{sacas} \mathrm{ha}^{-1}\right.$, em 2009, e 40,21 sacas ha-1 em 2011), em comparação ao ano de 2010 (32,18 sacas ha $\left.{ }^{-1}\right)$.

À densidade de 10.000 plantas ha ${ }^{-1}$, somente no ano de 2009, observou-se correlação entre o teor de $\mathrm{P}$ e a produtividade. Nesse ano, 74,91 sacas ha-1, o que está de acordo com a maior importância dos teores foliares de $\mathrm{P}$, em anos de alta produtividade, mencionada anteriormente, já que em 2010 e 2011, em que não houve correlação, possivelmente houve menor demanda de $\mathrm{P}$, em razão das produtividades menores $\left(48,09\right.$ sacas $a^{-1}$, em 2010, e 30,06 sacas por ha-1, em 2011). Altas produtividades estão associadas a maiores forças de dreno (Pereira et al., 2011), o que pode aumentar a importância do $\mathrm{P}$ na nutrição das plantas. Assim, em anos de baixa produtividade, menores disponibilidades já podem ser suficientes para atender a demanda dos drenos.
Nesta densidade, as lâminas de irrigação não se correlacionaram à produtividade, em nenhum dos três anos estudados (Tabela 3). A redução do espaçamento entre linhas e entre plantas reduz a produção por planta (Figueiredo et al., 2006), o que, de acordo com Rena et al. (1998), diminui a demanda por fotoassimilados e aumenta a exploração do solo pelas raízes. Esses fatores podem resultar em maior aproveitamento de água e nutrientes do solo pelas plantas. Assim, em lavouras tradicionais, a quantidade de água aplicada, nos diferentes manejos de irrigação, deve ser um fator mais determinante para a nutrição das plantas com P - com interferência direta no crescimento e na produtividade das plantas de cafeeiro - do que em lavouras adensadas.

As informações quanto aos mecanismos que regulam a interação entre Zn e P ainda são controversos (Reis Junior \& Martinez, 2002). No presente trabalho, em 2009, o teor foliar de Zn correlacionou-se positivamente com o de P, nas duas densidades de plantio avaliadas (Tabela 3). Nos anos posteriores, no entanto, essa correlação foi negativa, à exceção de 2011, quando não houve correlação à densidade de 10.000 plantas ha ${ }^{-1}$.

Tabela 3. Matriz de correlação entre as variáveis avaliadas em 2009, 2010 e 2011, à densidade de 3.333 (acima da diagonal) e de 10.000 plantas ha $^{-1}$ (abaixo da diagonal).

\begin{tabular}{lccccc}
\hline Fator & Lâmina & P foliar & Zn foliar & $\begin{array}{c}\text { Produti- } \\
\text { vidade }\end{array}$ & $\mathrm{AL}$ \\
\hline Lâmina & - & $0,85^{* *}$ & $0,51^{\text {ns }}$ & $0,83^{* *}$ & $0,98^{* * *}$ \\
P foliar & $0,94^{* *}$ & - & $0,85^{* *}$ & $0,80^{*}$ & $0,92^{* *}$ \\
Zn foliar & $0,90^{* *}$ & $0,91^{* *}$ & - & $0,73^{*}$ & $0,65^{\text {ns }}$ \\
Produtividade & $0,48^{\text {ns }}$ & $0,75^{*}$ & $0,61^{\text {ns }}$ & - & $0,88^{* *}$ \\
AL & $0,75^{*}$ & $0,88^{* *}$ & $0,93^{* *}$ & $0,83^{* *}$ & - \\
\hline & \multicolumn{5}{c}{ Ano 2010} \\
Lâmina & - & $0,93^{* *}$ & $-0,94^{* *}$ & $0,47^{\text {ns }}$ & $0,83^{* *}$ \\
P foliar & $0,83^{* *}$ & - & $-0,75^{*}$ & $-0,50^{\text {ns }}$ & $0,94^{* *}$ \\
Zn foliar & $-0,60^{\text {ns }}$ & $-0,81^{* *}$ & - & $0,52^{\text {ns }}$ & $-0,60^{\text {ns }}$ \\
Produtividade & $-0,25^{\text {ns }}$ & $-0,44^{\text {ns }}$ & $-0,12^{\text {ns }}$ & - & $-0,14^{\text {ns }}$ \\
AL & $0,93^{* *}$ & $0,97^{* * *}$ & $-0,73^{*}$ & $-0,42^{\text {ns }}$ & - \\
\hline \multirow{5}{*}{ Ano 2011} & & \\
Lâmina & - & $0,85^{* *}$ & $-0,37^{\text {ns }}$ & $0,94^{* *}$ & $0,89^{* *}$ \\
P foliar & $0,74^{*}$ & - & $-0,67^{*}$ & $0,88^{* *}$ & $0,93^{* *}$ \\
Zn foliar & $0,25^{\text {ns }}$ & $-0,46^{\text {ns }}$ & - & $-0,26^{\text {ns }}$ & $-0,75^{*}$ \\
Produtividade & $0,57^{\text {ns }}$ & $-0,04^{\text {ns }}$ & $0,87^{* *}$ & - & $0,79^{*}$ \\
AL & $0,90^{* *}$ & $0,42^{\text {ns }}$ & $0,61^{\text {ns }}$ & $0,86^{* *}$ & - \\
\hline
\end{tabular}

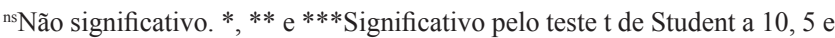
$1 \%$ de probabilidade, respectivamente. AL, área lateral do dossel. 
Problemas na nutrição com $\mathrm{Zn}$, com crescentes doses de P, têm sido relatados (Reis Junior \& Martinez, 2002). Além do possível efeito antagônico na absorção de Zn, maiores disponibilidades de $\mathrm{P}$ também podem causar o efeito de diluição, pelo aumento do desenvolvimento vegetativo (área lateral do dossel), o que também pode estar relacionado à diminuição do teor foliar de $\mathrm{Zn}$ (Guimarães et al., 2010; Crusciol et al., 2013).

Os teores foliares de $\mathrm{P}$ e $\mathrm{Zn}$ sofreram a interação dos efeitos das lâminas de irrigação e dos anos de cultivo, apenas na densidade de 3.333 plantas ha ${ }^{-1}$. Nesta densidade, durante os três anos de avaliação, o modelo linear positivo foi o que melhor descreveu o comportamento dos teores foliares de $\mathrm{P}$ em função das lâminas aplicadas (Figura 1), o que está de acordo com os resultados obtidos no estudo de correlação (Tabela 3). Para cada milímetro de água aplicado, houve aumento nos teores de P de: $0,0013 \mathrm{~g} \mathrm{~kg}^{-1}$, em 2009; 0,0004 g kg-1, em 2010; e 0,0007 $\mathrm{g} \mathrm{kg}^{-1}$, em 2011. Na média, mesmo no tratamento não irrigado os teores foliares de $\mathrm{P}$ foram maiores do que $1,2 \mathrm{~g} \mathrm{~kg}^{-1} \mathrm{e}$ situaram-se dentro da faixa de suficiência relatada por Guimarães et al. (1999) e Farnezi et al. (2009) - 0,18 dag $\mathrm{kg}^{-1}$ a 0,22 dag $\mathrm{kg}^{-1}$ - e acima do observado por Martinez et al. (2003) - 0,8 $\mathrm{g} \mathrm{kg}^{-1}$ a 2,0 $\mathrm{g} \mathrm{kg}^{-1}$. O solo utilizado apresentava teores suficientes de P (Tabela 1), o que explica os níveis adequados do nutriente nas plantas. À semelhança de Guerra et al. (2007), Reis et al. (2013) também observaram que, sob irrigação, o cafeeiro responde a adubações de até $400 \mathrm{~kg} \mathrm{ha}^{-1}$ de $\mathrm{P}_{2} \mathrm{O}_{5}$. Os autores concluem que, em condições de alta produtividade e alto suprimento de $\mathrm{P}$, as faixas de suficiência para esse nutriente devem ser ampliadas. Da mesma forma, os resultados do presente trabalho indicam que, com o uso de manejos com maiores lâminas, o aproveitamento do P pelas plantas aumenta, e o valor da faixa de suficiência de P é ampliado.

$\mathrm{O}$ teor foliar de $\mathrm{Zn}$ aumentou de forma linear com o aumento da lâmina de irrigação, em 2009; em 2010 e 2011, no entanto, houve tendência linear negativa, apesar dos baixos ajustes revelados pelos coeficientes de determinação (Figura 2). Essas tendências negativas são coerentes com os resultados obtidos para $\mathrm{o} P$ (Figura 1, Tabela 3) e alinham-se com a hipótese de efeito antagônico entre $\mathrm{P}$ e $\mathrm{Zn}$ em diversas culturas (Politi et al., 2013). Aquino et al. (2012), no entanto, lembram que essa relação antagônica ainda não é completamente conhecida.

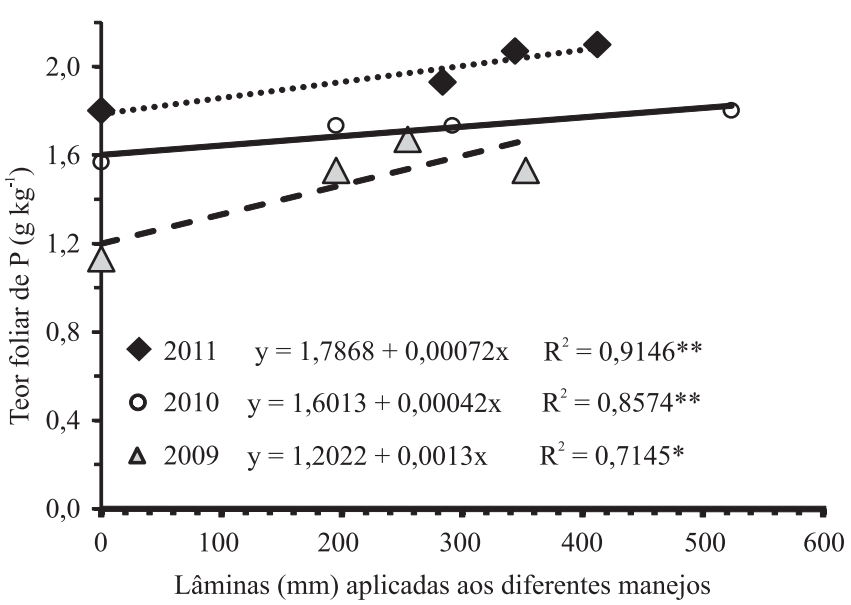

Figura 1. Teor foliar de fósforo em função das lâminas aplicadas de acordo com diferentes critérios de irrigação, ao longo de três anos, à densidade de plantas de $3.333 \mathrm{ha}^{-1}$.

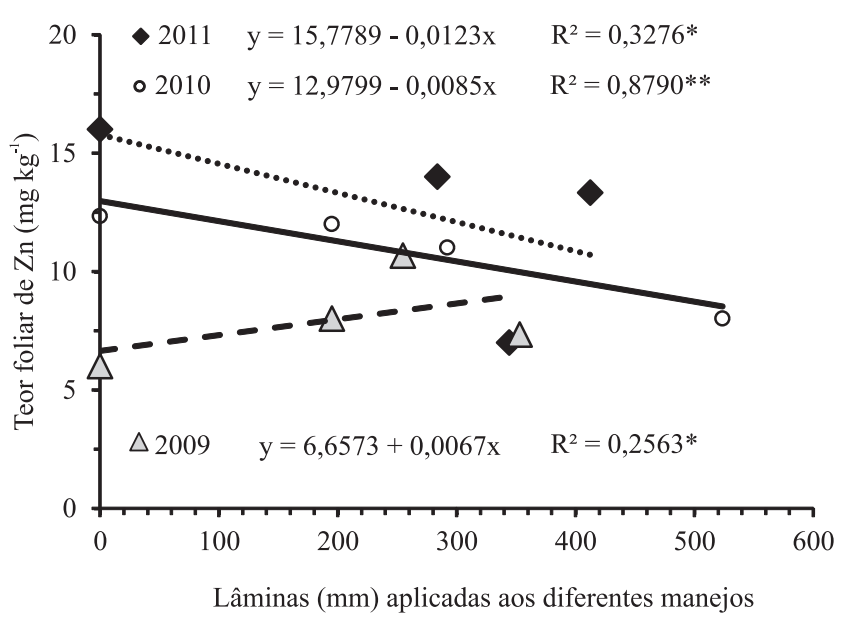

Figura 2. Teor foliar de zinco em função das lâminas aplicadas de acordo com diferentes critérios de irrigação, ao longo de três anos, à densidade de plantas de $3.333 \mathrm{ha}^{-1}$.

\section{Conclusões}

1. A irrigação de lavouras cafeeiras com maiores lâminas de irrigação favorece a absorção radicular de $\mathrm{P}$ e o crescimento vegetativo das plantas, tanto no sistema tradicional de cultivo como no adensado, bem como aumenta a produtividade em cultivos tradicionais.

2. A maior disponibilização de P pelo aumento da irrigação pode diminuir o teor de $\mathrm{Zn}$ nas folhas.

\section{Agradecimentos}

Ao Conselho Nacional de Desenvolvimento Científico e Tecnológico (CNPq), à Fundação de

Pesq. agropec. bras., Brasília, v.49, n.2, p.95-101, fev. 2014 DOI: 10.1590/S0100-204X2014000200003 
Amparo à Pesquisa de Minas Gerais (Fapemig) e ao Consórcio Pesquisa Café, pelo apoio financeiro e financiamento da pesquisa.

\section{Referências}

ALlEN, R.G.; PEREIRA, L.S.; RAES, D.; SMITH, M. Crop evapotranspiration: guidelines for computing crop water requirements. Rome: Food and Agriculture Organization of the United Nations, 1998. 300p. (FAO. Irrigation and drainage paper, $56)$.

AMARAL, J.F.T. do; MARTINEZ, H.E.P.; LAVIOLA, B.G.; TOMAZ, M.A.; FERNANDES FILHO, E.I.; CRUZ, C.D. Produtividade e eficiência de uso de nutrientes por cultivares de cafeeiro. Coffee Science, v.6, p.65-74, 2011.

AQUINO, L.A. de A.; AQUINO, R.F.B.A.; SILVA, T.C.; SANTOS, D.F. dos; BERGER, P.G. Aplicação do fósforo e da irrigação na absorção e exportação de nutrientes pelo algodoeiro. Revista Brasileira de Engenharia Agrícola e Ambiental, v.16, p.355-361, 2012. DOI: 10.1590/S1415-43662012000400004.

CRUSCIOL, C.A.C.; SOARATTO, R.P.; CASTRO, G.S.A.; COSTA, H.M. da; FERRARI NETO, J. Aplicação foliar de ácido silícico estabilizado na soja, feijão e amendoim. Revista Ciência Agronômica, v.44, p.404-410, 2013. DOI: 10.1590/ S1806-66902013000200025.

DANTAS, A.A.A.; CARVALHO, L.G. de; FERREIRA, E. Classificação e tendências climáticas em Lavras, MG. Ciência e Agrotecnologia, v.31, p.1862-1866, 2007. DOI: 10.1590/ S1413-70542007000600039.

FARNEZI, M.M. de M.; SILVA, E. de B.; GUIMARÃES, P.T.G. Diagnose nutricional de cafeeiros da região do Alto Jequitinhonha (MG): normas DRIS e faixas críticas de nutrientes. Revista Brasileira de Ciência do Solo, v.33, p.969-978, 2009. DOI: 10.1590/S0100-06832009000400021.

FAVARIN, J.L.; DOURADO NETO, D.; GARCÍA, A.G.; VILLA NOVA, N.A.; FAVARIN, M.G. da G.G. Equações para estimativa do índice de área foliar do cafeeiro. Pesquisa Agropecuária Brasileira, v.37, p.769-773, 2002. DOI: 10.1590/ S0100-204X2002000600005.

FERNANDES, A.L.T.; SANTINATO, R.; DRUMOND, L.C.D.; OLIVEIRA, C.B. de. Avaliação do uso de fertilizantes organominerais e químicos na fertirrigação do cafeeiro irrigado por gotejamento. Revista Brasileira de Engenharia Agrícola e Ambiental, v.11, p.159-166, 2007. DOI: 10.1590/ S1415-43662007000200005.

FIGUEIREDO, F.C.; FURTINI NETO, A.E.; GUIMARÃES, P.T.G.; SILVA, E. de B.; BOTREL, P.P. Eficiência da adubação com NPK na produção de cafezais adensados na região sul de Minas Gerais. Coffee Science, v.1, p.135-142, 2006.

GUERRA, A.F.; ROCHA, O.C.; RODRIGUES, G.C.; SANZONOWICZ, C.; RIBEIRO FILHO, G.C.; TOLEDO, P.M. dos R.; RIBEIRO, L.F. Sistema de produção de café irrigado: um novo enfoque. Item, v.73, p.52-61, 2007.
GUIMARÃES, P.T.G.; GARCIA, A.W.R.; ALVAREZ V., V.H.; PREZOTTI, L.C.; VIANA, A.S.; MIGUEL, A.E.; MALAVOLTA, E.; CORRÊA, J.B.; LOPES, A.S.; NOGUEIRA, F.D.; MONTEIRO, A.V.C. Cafeeiro. In: RIBEIRO, A.C.; GUIMARÃES, P.T.G.; ALVAREZ V., V.H. (Ed.). Recomendação para o uso de corretivos e fertilizantes em Minas Gerais: 5a aproximação. Viçosa: Comissão de Fertilidade do Solo do Estado de Minas Gerais, 1999. p.289-302.

GUIMARÃES, R.J.; SCALCO, M.S.; COLOMBO, A.; ASSIS, G.A.; CARVALHO, G.R.; ALEXANDRE, L.P.B. Adubação para primeiro ano pós plantio $\left(\mathrm{N} \mathrm{e}_{2} \mathrm{O}\right)$ de cafeeiros fertirrigados na região sul de Minas Gerais. Coffee Science, v.5, p.137-147, 2010.

MARTINEZ, H.E.P.; MENEZES, J.F.S.; SOUZA, R.B. de; VENEGAS, V.H.A.; GUIMARÃES, P.T.G. Faixas críticas de concentrações de nutrientes e avaliação do estado nutricional de cafeeiros em quatro regiões de Minas Gerais. Pesquisa Agropecuária Brasileira, v.38, p.703-713, 2003. DOI: 10.1590/ S0100-204X2003000600006.

MERA, A.C.; OLIVEIRA, C.A. da S.; GUERRA, A.F.; RODRIGUES, G.C. Regimes hídricos e doses de fósforo em cafeeiro. Bragantia, v.70, p.302-311, 2011.

MIRANDA, W.L.; GUIMARÃES, R.J.; MAGALHÃES, P.B.; COLOMBO, A.; OLIVEIRA, P.M. de. Desenvolvimento vegetativo de plantas de café arábica enxertadas sobre café robusta e submetidas à reposição hídrica. Pesquisa Agropecuária Brasileira, v.46, p.1618-1624, 2011. DOI: 10.1590/S0100-204X2011001200006.

MANTOVANI, E.C.; BERNARDO, S.; PALARETTI, L.F. Irrigação: princípios e métodos. Viçosa: Ed. da UFV, 2007. 358p.

PEREIRA, S.P.; BARTHOLO, G.F.; BALIZA, D.P.; SOBREIRA, F.M.; GUIMARÃES, R.J. Crescimento, produtividade e bienalidade do cafeeiro em função do espaçamento de cultivo. Pesquisa Agropecuária Brasileira, v.46, p.152-160, 2011. DOI: 10.1590/S0100-204X2011000200006.

POLITI, L.S.; FLORES, R.A.; SILVA, J.A.S. da; WADT, P.G.S.; PINTO, P.A. da C.; PRADO, R. de M. Estado nutricional de mangueiras determinado pelos métodos DRIS e CND. Revista Brasileira de Engenharia Agrícola e Ambiental, v.17, p.11-18, 2013. DOI: $10.1590 / \mathrm{S} 1415-43662013000100002$.

REIS JUNIOR, R. dos A.; MARTINEZ, H.E.P. Adição de Zn e absorção, translocação e utilização de $Z n$ e $P$ por cultivares de cafeeiro. Scientia Agricola, v.59, p.537-542, 2002. DOI: 10.1590/ S0103-90162002000300019.

REIS, T.H.P.; FURTINI NETO, A.E.; GUIMARÃES, P.T.G.; GUERRA, A.F.; OLIVEIRA, C.H.C. de. Estado nutricional e frações foliares de P no cafeeiro em função da adubação fosfatada. Pesquisa Agropecuária Brasileira, v.48, p.765-773, 2013. DOI: 10.1590/S0100-204X2013000700009.

RENA, A.B.; NACIF, A. de P.; GUIMARÃES, P.T.G.; PEREIRA, A.A. Poda do cafeeiro: aspectos morfológicos ecofisiológicos e agronômicos. Informe Agropecuário, v.19, p.61-70, 1998.

REZENDE, R.; HELBER JÚNIOR, C.; SOUZA, R.S. de; ANTUNES, F.M.; FRIZZONE, J.A. Crescimento inicial de duas cultivares de cafeeiro em diferentes regimes hídricos e dosagens de fertirrigação. Engenharia Agrícola, v.30, p.447-458, 2010. DOI: 10.1590/S0100-69162010000300009. 
SANTINATO, R.; FERNANDES, A.L.T. Cultivo do cafeeiro irrigado em plantio circular sob pivô central. Belo Horizonte: O Lutador, 2002. 252p.

SANTOS, H.G. dos; JACOMINE, P.K.T.; ANJOS, L.H.C. dos; OLIVEIRA, V.A. de; OLIVEIRA, J.B. de; COELHO, M.R.; LUMBRERAS, J.F.; CUNHA, T.J.F. (Ed.). Sistema brasileiro de classificação de solos. 2.ed. Rio de Janeiro: Embrapa Solos, 2006. $306 \mathrm{p}$.

SCALCO, M.S.; ALVARENGA, L.A.; GUIMARÃES, R.J.; COLOMBO, A.; ASSIS, G.A. Cultivo irrigado e não irrigado do cafeeiro (Coffea arabica L.) em plantio superadensado. Coffee Science, v.6, p.193-202, 2011.

SERRA, E.L.; SCALCO, M.S.; GUIMARÃES, R.J.; COLOMBO, A.; MORAIS, A.R. de; CARVALHO, C.H.M. de. Funções de produção do cafeeiro irrigado em diferentes densidades de plantio. Coffee Science, v.8, p.157-165, 2013.

SILVA, C.A. da.; TEODORO, R.E.F.; MELO, B. de. Produtividade e rendimento do cafeeiro submetido a lâminas de irrigação. Pesquisa Agropecuária Brasileira, v.43, p.387-394, 2008. DOI: 10.1590/S0100-204X2008000300014.

SOBREIRA, F.M.; GUIMARÃES, R.J.; COLOMBO, A.; SCALCO, M.S.; CARVALHO, J.G. Adubação nitrogenada e potássica de cafeeiro fertirrigado na fase de formação, em plantio adensado. Pesquisa Agropecuária Brasileira, v.46, p.9-16, 2011. DOI: $10.1590 /$ S0100-204X2011000100002.

VAN GENUCHTEN, M.T. A closed-form equation for prediting the hydraulic conductivity of unsaturated soils. Soil Science Society of America Journal, v.44, p.892-898, 1980. DOI: 10.2136/sssaj1980 $.03615995004400050002 x$.

Recebido em 8 de abril de 2013 e aprovado em 30 de janeiro de 2014 